\title{
Community-based wound care in the Netherlands: Implementing a regional network for wound care
}

\author{
The transition of hospital care to primary care is one of the mainstays \\ of national health care policy in the Netherlands. This paper presents the \\ results of a successful initiative to establish a regional community-based \\ wound care network in the Zwolle region of the Netherlands.
}

Keywords:

community-based; complex wounds; network medicine; nurse practitioner; organisational innovation; primary care; regional network; wound care team; wound expertise centre

\begin{abstract}
Background

The transition of hospital care to primary care is one of the mainstays of national health care policy in the Netherlands. After introducing an innovative new concept of wound care in 2015, this successful initiative expanded to a regional community-based wound care network in the Zwolle region.

\section{Method}

A descriptive analysis of several outcome parameters was performed on prospectively retrieved data. In the period 2018-2019, a weekly consultation hour was started after installing a local team in six primary care practices. The team included a nurse practitioner specialised in wound care, general practitioner assistants and a general practitioner. An experienced surgeon from the regional hospital could be consulted via telemedicine and visited the practices regularly.
\end{abstract}

\section{Findings}

In total, 869 patients were seen; among them, 695 patients had 814 wounds. Most of these wounds (73\%) were located on a lower extremity. The program treated mainly older patients with moderate or severe co-morbidities; $91 \%$ were served in a primary care practice. An access time of up to six weeks was reported for $80 \%$ of patients. A healing rate of $91 \%$ was achieved in 417 wound patients treated by the nurse practitioner. In the end, $9 \%$ of the cases were referred to the regional hospital.

\section{Conclusions}

Almost all patients with wound-related problems can be served by a local nurse practitioner-led team. It seems that the Zwolle regional wound care network has adopted the national policy goals, such as short access times and more preventative measures, with high rates of wound healing and a minimum number of referrals.

In the Netherlands, national guidelines were developed in 2018 with the goal of implementing basic conditions to ensure good care outcomes and a good organisation of wound care. ${ }^{1}$ These guidelines state that wound care comprises more than the application of bandages or medical supplies by paramedics; instead, wound care is a medical domain that needs to be performed with medical expertise by well-trained care professionals. In this view, doctors should most- 
ly facilitate other wound professionals' work; their primary responsibility is ensuing that quality care is being provided. The purpose of the guidelines is to deliver wound care closer to the patient's community and to realise a decrease in the number of referrals to the hospital, thus minimising health care expenditures.

We have already reported that, in the Netherlands, intensive and complex wound care can be performed in local general practices with good outcomes and at minimal costs. ${ }^{2}$ The outcomes were so positive that we decided to implement the model on a wider regional scale. One of the advantages of the care concept is that fewer patients are referred to hospital care, because they can receive almost all the wound care they need in their own community. The wound care is performed by a nurse practitioner who specialises in wounds, working in co-operation with a local team of general practitioners and general practitioner assistants. The care model that functions locally can be linked together perfectly to a regional wound care network. Thus, high quality wound care is within reach of a local community, with the best clinical results and at the lowest cost. ${ }^{3}$

\section{Key messages}

- This paper presents the results of a successful initiative to establish a regional community-based wound care network in the Zwolle region of the Netherlands. The cohort study's organisation, set up and requirements are outlined. Data were collected concerning patients' age, sex, wound location, wound age, wound healing, comorbidities, outcomes of consultation and aspects of their referral to the hospital.

- The aim of this paper is to share the results of efforts to organise wound care in a general practice setting and to demonstrate the benefits for patients and the Dutch health care system alike.

- By organising wound care in a general practice setting managed by a local team headed by a nurse practitioner who specialises in wound care, about $90 \%$ of the wounds healed without a referral to the regional hospital.

- Access time was less than six weeks for $80 \%$ of the patients; mainly older patients with moderate or severe co-morbidities were treated.

\section{METHOD}

In 2018, we commenced a weekly consultation hour for primary care practices in the Zwolle region of the Netherlands. Between February 2018 and September 2019, six general practitioner centres participated in the wound care network. These six practices served a population of about 53,000 people as of May 2020. We performed a cohort study in which prospectively retrieved data were used. A descriptive analysis was performed related to patients' age, sex, wound location, wound age, wound healing, comorbidities, outcomes of consultation and aspects of their referral to a hospital.

A local wound team was established and composed of a nurse practitioner, general practitioner assistants and a general practitioner. A strong collaboration with local home-care service nurses was also established from the beginning. A surgeon from the Wound Expertise Centre (WEC) Isala Zwolle could be consulted via telemedicine. Surgeons from the WEC visited the local wound teams every six weeks to participate in consultations regarding more complex cases at the site, in order to minimise referrals to the hospital. There were no other locally involved medical specialties. The nurse practitioner could also visit patients in their homes, if they were unable to come to the general practice. The local wound team also cooperated with other primary care professionals, including physical therapists, oedema therapists, chiropodists, podiatrists or an orthopaedic shoemaker. Only when necessary were patients referred to the hospital for specific diagnostic purposes or treatments, such as the application of lower limb casts or operative treatments.

The wound teams reported all relevant information via electronic patient files. During the consultation hour, the nurse practitioner could make use of all instruments to perform surgical debridement. Anklebrachial-index and toe-pressure were performed with an automated kit. Additional diagnostics used by the nurse practitioner concerned skin biopsies, wound swabs or blood tests. Radiological diagnostics could be ordered at the hospital in Zwolle, so that the local wound team maintained its leadership over the case. Wound photos taken with a 2D-camera and particular dimensions of the wound were also measured every week.

Since the nurse practitioner was the only wound careeducated member of the team, a specific education 
programme was the mainstay of the implementation strategy. All local wound teams were trained by the nurse practitioner in wound biology, diagnostics and treatments, such as debridement and compression therapy. Educational materials were developed in collaboration with the WEC Isala. Furthermore, all general practitioner assistants in the Zwolle region were trained four times per year in a regional meeting led by wound professionals from the WEC. The general practitioner did not follow a specific education programme, but learned by doing while collaborating weekly with the nurse practitioner. General practitioners also participated when the surgeon was visiting the practice, every 4-6 weeks. Wound-educated nurses employed by the home care services were also invited to join the educational programme locally.

A special wound electonic medical record (EMR) system, along with process-information technology and data models, was developed for the project, and the data were entered using specially developed software. The relevant information was discussed every eight weeks in a special meeting with the WEC and the nurse practitioners. The general practice management and local wound team were visited twice annually by the project leaders to evaluate and discuss management information and the outcomes of the wound care provided. In addition, costs related to the outcomes of care were also monitored, in order to quantify the added value of the care process. The number and duration of visits, home visits, specific interventions performed and bandages and medical supplies used were registered.

Reimbursement was based on a production-related system, not on a patient-dependent budget. The pay-

Table 1.

Localisation of 815 wounds

\begin{tabular}{lccc}
\hline Location & Left & Right & Total \\
\hline Other & $14 \%$ & $13 \%$ & $27 \%$ \\
\hline Upper leg & $1 \%$ & $2 \%$ & $3 \%$ \\
\hline Lower leg & $18 \%$ & $17 \%$ & $35 \%$ \\
\hline Ankle & $3 \%$ & $3 \%$ & $7 \%$ \\
\hline Heel & $2 \%$ & $2 \%$ & $5 \%$ \\
\hline Midfoot & $1 \%$ & $1 \%$ & $2 \%$ \\
\hline Forefoot & $3 \%$ & $3 \%$ & $6 \%$ \\
\hline Toes & $8 \%$ & $9 \%$ & $16 \%$ \\
\hline Total & $\mathbf{5 1 \%}$ & $\mathbf{4 9} \%$ & \\
\hline
\end{tabular}

Figure 1.

Distribution of patients by age and sex $(\mathrm{n}=869)$

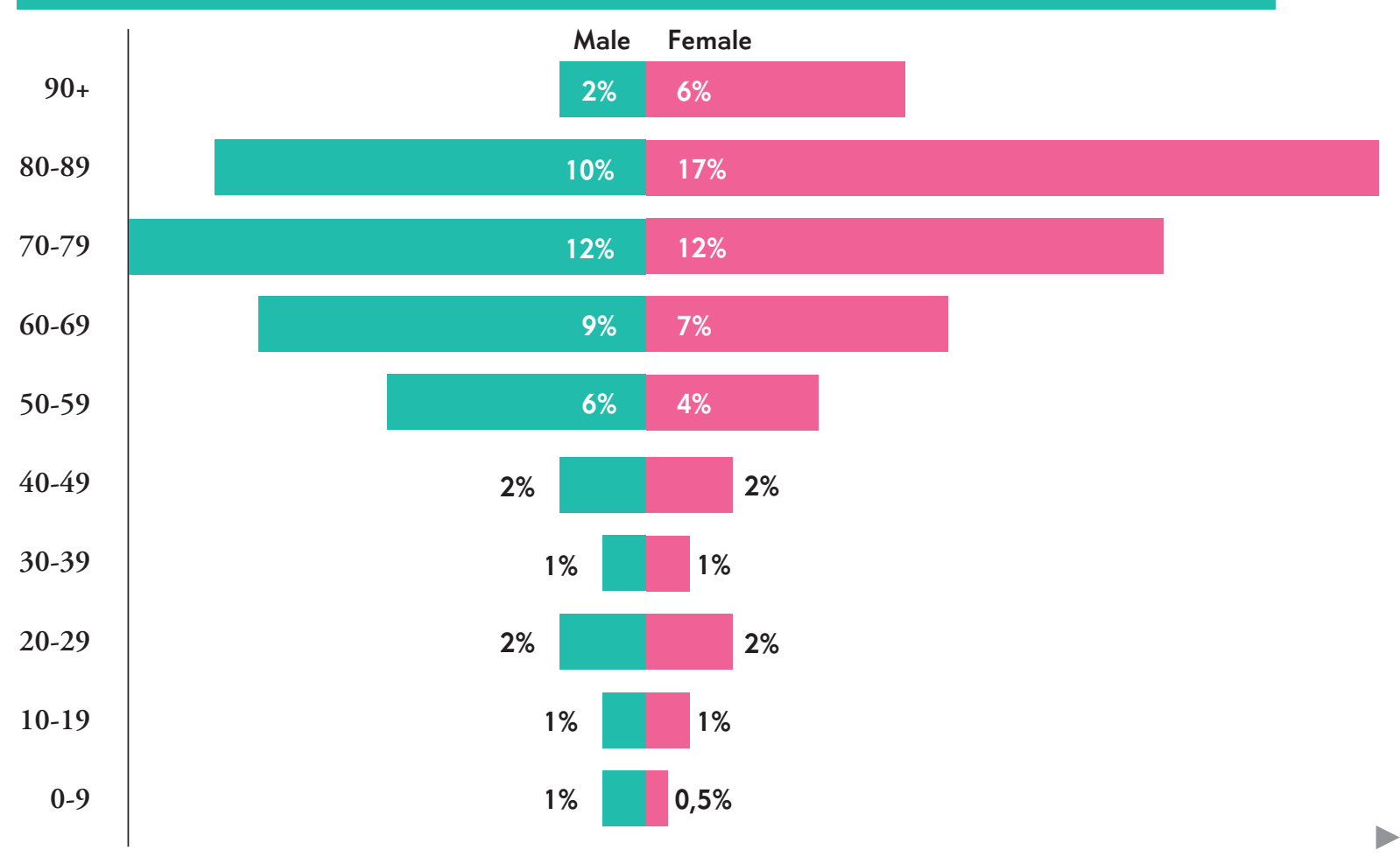


Table 2.

Distribution of the absolute number of registered secondary diagnoses per

location in a total of 869 patients. Note that patients may suffer from more than

one secondary diagnosis.

Foot $n=252$, Lower $\operatorname{leg} n=365$, Upper leg $n=26$, Other locations $n=226$

\begin{tabular}{lccccc}
\hline Diagnosis & \multicolumn{2}{c}{ Location } & \multicolumn{2}{c}{ Total } \\
& Foot & Lower leg & Upper leg & Other & \\
\hline Diabetes & 88 & 54 & 3 & 40 & 185 \\
\hline PAOD & 56 & 43 & 3 & 13 & 115 \\
\hline Venous disease & 58 & 99 & 4 & 20 & 181 \\
\hline Heart disease & 164 & 171 & 12 & 104 & 451 \\
\hline Neurological disease & 50 & 37 & 3 & 26 & 116 \\
\hline Lung disease & 33 & 52 & 4 & 33 & 122 \\
\hline Renal disease & 53 & 44 & 2 & 16 & 115 \\
\hline Malignancy & 30 & 30 & 5 & 26 & 91 \\
\hline Immune-deficiency & 28 & 35 & 2 & 13 & 78 \\
\hline Total & $\mathbf{5 6 0}$ & $\mathbf{5 6 5}$ & $\mathbf{3 8}$ & $\mathbf{2 9 1}$ & $\mathbf{1 4 5 4}$ \\
\hline
\end{tabular}

PAOD, Peripheral Arterial Occlusive Disease

ments for, respectively, the general practise, the nurse practitioner, the consulting surgeon and home care services were all made separately. Particular financial settlements and project subsidies were arranged by the regional insurance company in cooperation with the regional hospital for the duration of the programme's three years.

\section{RESULTS}

A total of 869 patients were seen by the local wound teams during the observation period. This means that about one percent $(1.3 \%)$ of the local population received treatment for a wound-related problem during the study period. The 869 people together had a total of 814 wounds. Figure 1 shows that the greatest number of patients were over 50 years of age. The male-female distribution was about equal.

The localisation of the wounds can be seen in Table 1 . What stands out is that $42 \%$ of the wounds were localised to the lower leg, while about $29 \%$ had a foot wound. In the foot, the toes were most often affected. The category 'other wounds' (27\%) comprised wounds of the head, arms, trunk or buttocks. Pressure sores were seen only in 17 patients (2\%).

The distribution of secondary diagnoses in relation to location is displayed in Table 2. Cardiac-related disorders were, by far, in the majority and concerned $31 \%$ of all diagnoses. Heart disease predominated mainly in foot and lower leg wounds. Venous disease was also manifest in lower leg ulcers, while all the other disorders were equally distributed. Diabetes was present
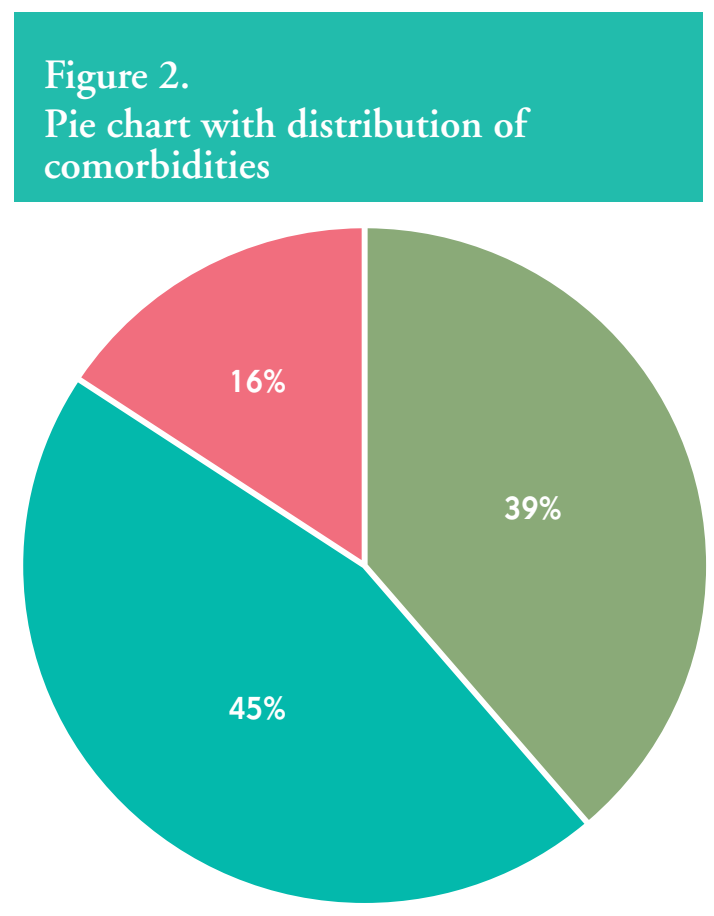

None

Mild

Severe

Note: 'None' means no comorbidity, 'Mild' means 1 or 2 secondary diagnoses, 'Severe' means 3 or more secondary diagnoses. 
Figure 3.

Distribution of wound age, from the origin of the wound $(n=815)$
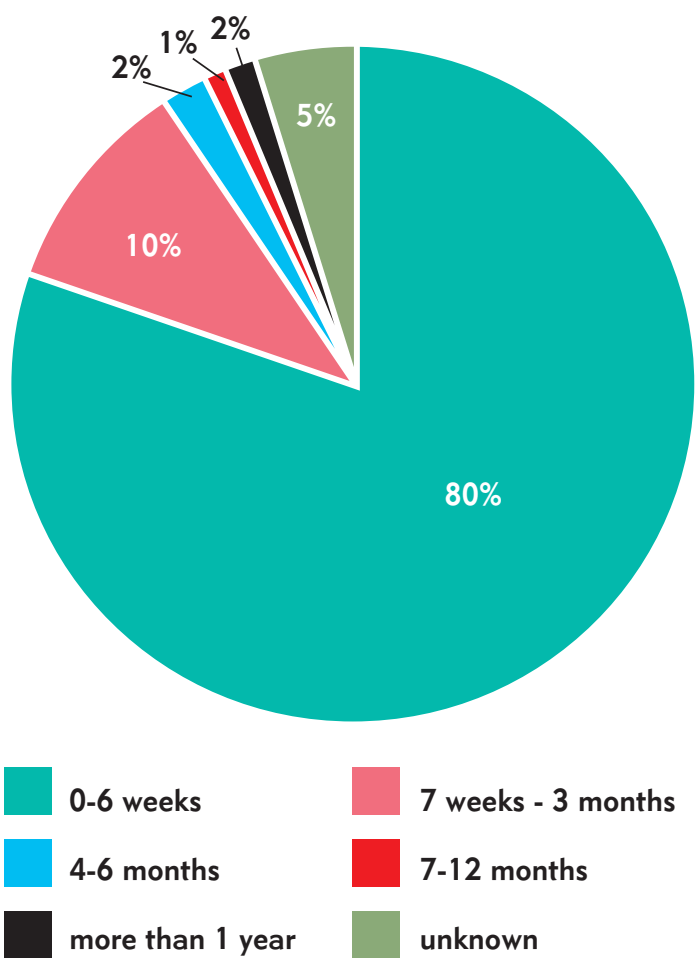

$0-6$ weeks

4-6 months

more than 1 year

7 weeks - 3 months

in patients with foot and lower leg wounds. Diabetes was present in about one third of foot wounds (35\%). Peripheral arterial occlusive disease was associated with foot wounds in $22 \%$ of cases, but it was also present in $12 \%$ of lower leg wounds.

An overview of the severity of related co-morbidities can be seen in Figure 2. Roughly 39\% of the patients were healthy and had no secondary diagnosis, while all other patients did have a co-morbidity (61\%). A substantial group, $16 \%$, had three or more secondary diagnoses and suffered from a severe co-morbidity.

Figure 3 shows that $80 \%$ of the patients came to the wound consultation hour within six weeks of the origination of their wound-related problem. Almost all $(90 \%)$ of the patients visited the wound team within a period less than three months. In only $5 \%$ of patients had the wound been present for more than six months.

In total, 695 patients with a wound problem were seen, and in a selection of 417 (61\%) of more complex cases, both diagnosis and wound treatment were performed under supervision of the nursing prac-
Figure 4

Overview of outcomes of consultations performed by the community wound team in the primary care setting $(\mathrm{n}=869$ patients)

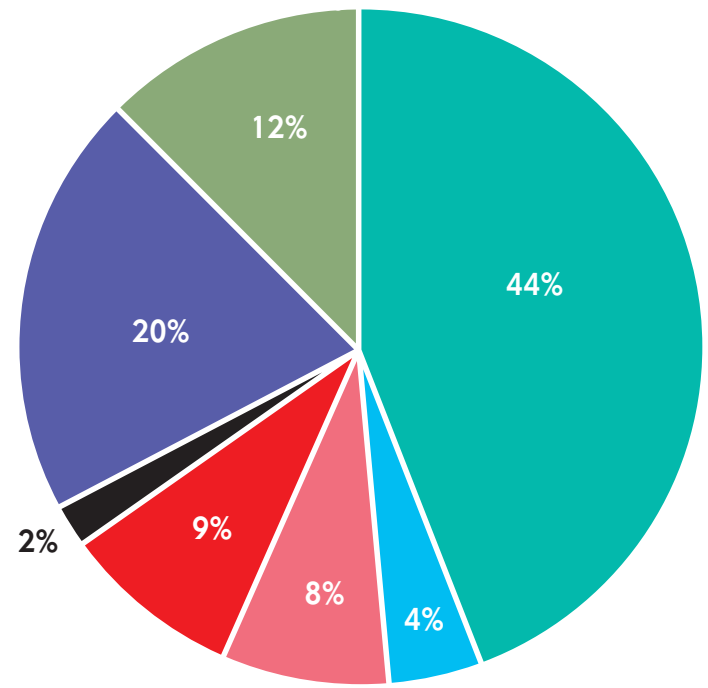

Wound healed solely in our primary care setting

Chronic wound treated solely in our primary care setting

Referred - to other primary care

Refferred - to hospital

Patient passed away

Only preventive care solely in our primary care setting

Not (yet) known

titioner. Eventually, complete healing was achieved in 397 patients (healing rate: 91\%). In 9\% of cases, the wound remained chronic, but progress was made leading to fewer complaints or less discomfort. In $28 \%$ of the 869 patients, the local wound team only performed diagnostic activities or proposed a plan for treatment. Further care was performed by colleague primary care partners in the community. The group with an unknown consultation outcome $(12 \%)$ refers to those patients who were not registered with the concerning general care practice, or who were later seen by other care professionals.

Referral to the WEC was eventually deemed necessary in $9 \%$ of patients (see also Table 3). A majority of referrals to the WEC concerned foot wounds. Additional diagnostic procedures, such as duplex ultrasounds or complex radiologic tests, were done. Most 
Table 3.

Overview of the 71 patients referred to the multidisciplinary

Wound Expertise Centre at Isala Hospital.

\begin{tabular}{|c|c|c|c|c|c|}
\hline & Foot & Lower leg & Upper leg & Other & Total \\
\hline Wound Expertise Centre & 24 & 19 & 3 & 5 & 51 \\
\hline Hospital Admissions & 15 & 4 & - & 1 & 20 \\
\hline Vascular intervention & 12 & 2 & - & - & \\
\hline Minor amputation & 11 & - & - & - & \\
\hline Conservative & 3 & 2 & - & 1 & \\
\hline Major amputation & - & - & - & - & \\
\hline Wounds Healed & 33 & 18 & 2 & 3 & 56 \\
\hline
\end{tabular}

lower leg patients were referred to dermatologists to analyse and treat ulcers for vasculitis or other specific dermatological diagnoses. Hospital admission was necessary in 20 patients. The main goal of treatment was conservative infection treatment or limb salvage by performing vascular procedures or foot surgery. Fortunately, no major amputations have been performed to date.

\section{DISCUSSION}

We report on the experiences with this programme two years after we formed a regional network for complex wound care in the region of the Dutch city of Zwolle (450,000 inhabitants). The project began in 2018 in the primary care setting for about 33,000 residents of three local communities. At present, we have expanded to six towns and serve a population of 53,000 people. The results show that more than $90 \%$ of patients could be treated in the primary care setting with a high quality outcome. Many patients were elderly people with lower extremity wounds, and most were seen within six weeks after the initiation of the wound. We know from earlier reports that, in the Netherlands, the average access time for wound care is more than 30 weeks. ${ }^{4}$ The local team was able to serve more than $90 \%$ of patients in the community. With this in mind, we can conclude that we have already improved the accessibility of wound care in the Zwolle region.
Another positive observation we made is that the number of referrals to the hospital was just $9 \%$. This is a number three times lower than the Dutch average of $30 \%$ mentioned in a national report dating from 2014. ${ }^{4}$ Still, most wounds healed in the hospital setting with only a minimum of hospitalisation and invasive interventions. The healing rate of selected wounds was $91 \%$ with the local wound team and $79 \%$ in the hospital setting. This is a rate above the international standard of $75 \%$ for wound healing. In conclusion, it appears that the quality indicators for outcomes of wound care are at a good level in our region.

The first step to establishing a working wound care service was the formation of local dedicated wound teams directed by the regional WEC. The nurse practitioner fulfils a central role in the team as a 'wound navigator. ${ }^{5}$ This professional was trained in complex wound care in the WEC and has more knowledge of wounds than is available from generalist professionals locally. Together with the general practitioner, the nurse practitioner could shape the medical policy and ensure access to the WEC. Furthermore, the nurse practitioner linked other local care professionals together to an organised wound team. The medical leadership, education and quality guarantee were taken care of in the regional WEC. In fact, the network may be considered a virtual multi-professional and multidisciplinary wound team, a notion proposed by EWMA in $2014 .{ }^{5}$ 
It has already been discussed in the EWMA community that good working wound care stands on three prerequisites, financial preconditions, educational preconditions and organisational preconditions. ${ }^{5}$ In our opinion, the most important message of the EWMA position paper is that modern care for wounds demands wound education and sound therapeutic skills organised in multidisciplinary and multiprofessional teams. The latter may imply that wound care is not best performed by generalist health care professionals in primary care or home care services. Therefore, we came up with the concept of introducing wound-educated nurse practitioners in primary practice settings to strengthen wound care in the community. In this paper, we discuss how we dealt with system constraints and we addressed the three prerequisites mentioned above. This study shows that considerable quality improvement can be achieved with a local team in which the nurse practitioner is the only wound-educated professional. Thus, this network succeeded in pursuing the best achievable level of wound care in the Dutch health care system.

\section{CONCLUSION}

The most important finding of this study is that almost all patients with wound-related problems can be served by a local team in their own community. Furthermore, introducing a wound-trained nurse practitioner in a local team leads to good quality wound care, not only for patients suffering from wounds but also in terms of preventive care. Moreover, the results provide epidemiologic insight into wound- healing rates within primary care practices. It seems that the Zwolle regional wound care network established national policy goals, such as short access time and more preventative measures, which led to high rates of wound-healing and a minimum of referrals to the hospital.

\section{Recommendations for clinical practice}

For future success and the securing of other regional wound network initiatives, we think that future investments and research should focus on:

- Educating nurse practitioners and local caregivers for the wound team

- A specific information and communication technologies (ICT data) model to monitor outcomes and costs, to optimise health economic value in the whole chain of wound care

- A new financial model that covers all care activities and medical supplies, to ensure fair reimbursement

- Specific e-health applications to support wound patients with self-management and empowerment

\section{Acknowledgements}

We gratefully thank Mr Pieter van den Berg and Mrs Marja Zwaan for their advice and support while implementing the regional network.

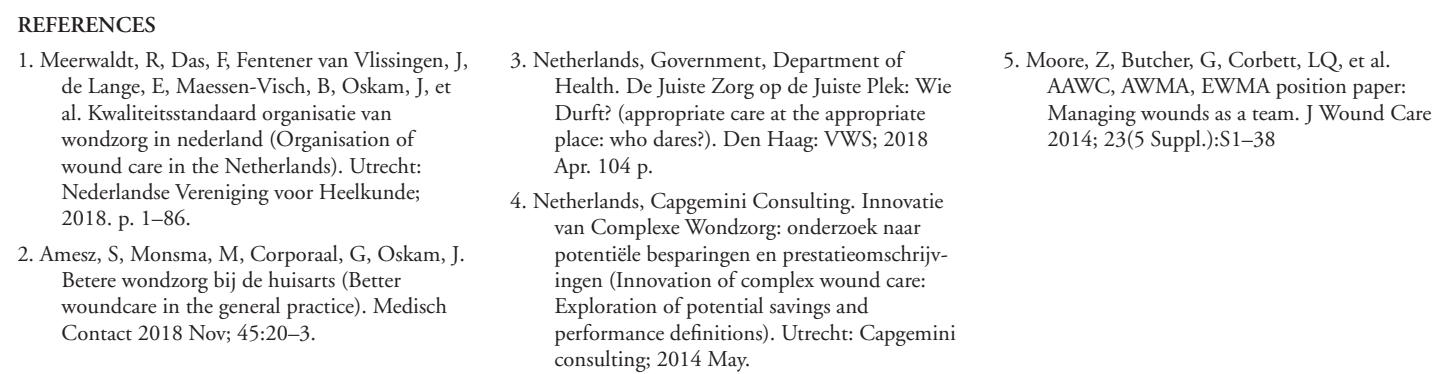

3. Netherlands, Government, Department of Health. De Juiste Zorg op de Juiste Plek: Wie Durft? (appropriate care at the appropriate place: who dares?). Den Haag: VWS; 2018 Apr. 104 p.

4. Netherlands, Capgemini Consulting. Innovatie van Complexe Wondzorg: onderzoek naar potentiële besparingen en prestatieomschrijvingen (Innovation of complex wound care: Exploration of potential savings and performance definitions). Utrecht: Capgemini consulting; 2014 May. 